\title{
Effects of somatosensory stimulation on corticomotor excitability in patients with unilateral cerebellar infarcts and healthy subjects - preliminary results
}

Suzete Nascimento Farias da Guarda ${ }^{1,2,4^{*}}$ and Adriana Bastos Conforto ${ }^{1,3}$

\begin{abstract}
Background: In healthy humans, somatosensory stimulation in the form of 2 h-repetitive peripheral afferent nerve stimulation (SS) increases excitability of the contralateral motor cortex. In this preliminary study, we explored effects of SS on excitability to transcranial magnetic stimulation (TMS) in patients with unilateral cerebellar infarcts and age-matched controls.

Methods: Ten patients with infarcts in one cerebellar hemisphere and six age-matched controls participated in the study. Each subject participated in one session of active, and one session of sham SS delivered to the median nerve ipsilateral to the cerebellar infarct in patients, and to the homologous nerve in controls. Before and after each session, the following TMS measures were performed: resting motor threshold (rMT), motor evoked potentials (MEPs), short-interval intracortical inhibition (SICl) and short-interval intracortical facilitation (SICF). Amplitudes of motor evoked potentials were normalized to amplitudes of supramaximal M responses (MEP/M ratios).
\end{abstract}

Results: In the control group, there was a significant increase in $\mathrm{rMT}$, and a significant increase in MEP/M ratios after active, but not after sham SS. There were no significant differences in rMT or MEP/M ratios in the group of patients after active or sham SS. There were no significant differences in SICI or SICF after active or sham SS in either group.

Conclusion: Consistent with results reported in rodents, these preliminary findings suggest for the first time in humans, that normal cerebellar activity is required so that SS can modulate excitability of the sensorimotor cortex.

Keywords: Stroke, Somatosensory stimulation, Transcranial magnetic stimulation

\section{Background}

In rodents, somatosensory stimulation (SS) enhances excitability of the contralateral motor cortex. This effect is decreased by down-regulation of cerebellar function, indicating that responsiveness to SS can be modulated by the cerebellum [1-4].

In healthy humans, SS in the form of $2 \mathrm{~h}$-repetitive peripheral afferent nerve stimulation increases excitability of the contralateral motor cortex, reflected by increase in amplitudes of motor potentials evoked by transcranial magnetic stimulation (TMS) $[5,6]$.

In both animals and humans, there is evidence that the effect of SS on cortical excitability is mediated at a supraspinal level [6-8], but the exact neural structures and pathways involved are still unclear. We explored effects of SS in the form of median nerve stimulation, in patients with infarcts affecting one cerebellar hemisphere, and in age-matched controls.

\section{Results}

Before and after one session of active or sham SS (Figure 1), the following TMS measures were performed: resting motor threshold (rMT), motor evoked potentials (MEPs),

${ }^{1}$ Hospital das Clínicas/São Paulo University, São Paulo, Brazil

${ }^{2}$ Hospital São Rafael, Salvador, Brazil

Full list of author information is available at the end of the article 


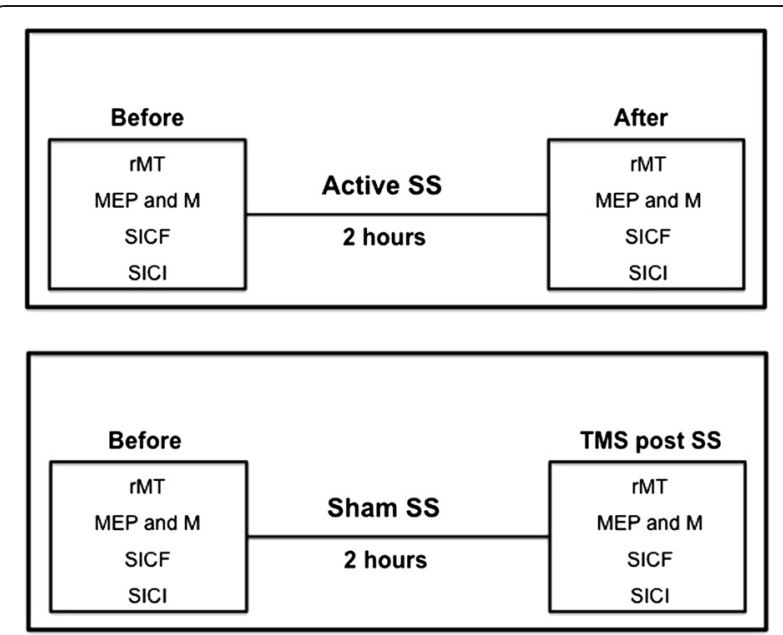

Figure 1 Experimental design. Each patient was submitted to two separate experimental sessions (active somatosensory stimulation, Active SS; sham somatosensory stimulation, Sham SS). In both sessions, resting motor thresholds (rMT), motor evoked potentials (MEPs), short-interval intracortical facilitation (SICF), short-interval intracortical inhibition $(\mathrm{SICl})$ and supramaximal $\mathrm{M}$ response amplitudes were measured before and after somatosensory stimulation.

short-interval intracortical inhibition (SICI) and shortinterval intracortical facilitation (SICF). Amplitudes of motor evoked potentials were normalized to amplitudes of supramaximal $\mathrm{M}$ responses (MEP/M ratios).

Table 1 shows results of rMT, SICI and SICF.

All subjects reported intense paresthesias in the median nerve territory during the 2-h period of active SS, and none reported paresthesias during the session of sham SS. Table 2 shows initial stimulus intensities in each patient. Initial stimulation intensities were (average \pm standard deviation), $48.5 \pm 24.8$ Volts $(\mathrm{V})$ in patients and $64.2 \pm 9.3 \mathrm{~V}$ in controls. There was no significant difference in initial intensities between the two groups $(\mathrm{p}=0.368)$.

Table 1 Corticomotor excitability in patients and controls before and after active and sham sessions

\begin{tabular}{lllll}
\hline Measure & Pre active & Post active & Pre sham & Post sham \\
\hline Patients & & & & \\
rMT (\%s.o.) & $50.7 \pm 10.8$ & $51.2 \pm 11.1$ & $51.4 \pm 11.8$ & $51.3 \pm 12.2$ \\
SICI (\%) & $85.1 \pm 81.2$ & $70.4 \pm 41.4$ & $64.2 \pm 23.7$ & $82 \pm 53.8$ \\
SICF (\%) & $181.8 \pm 107.4$ & $166.3 \pm 43.4$ & $152.8 \pm 56.4$ & $177.9 \pm 78.2$ \\
Controls & & & & \\
rMT (\%s.o.) & $51.5 \pm 8.5$ & $52.7 \pm 8.7^{*}$ & $50 \pm 6.7$ & $49.2 \pm 7.7$ \\
SICI (\%) & $49 \pm 27.7$ & $58.8 \pm 24.8$ & $38.8 \pm 24.5$ & $46.9 \pm 35$ \\
SICF (\%) & $204.2 \pm 91.1$ & $135.2 \pm 62.3$ & $202.8 \pm 128.8$ & $155 \pm 52.5$ \\
\hline
\end{tabular}

rMT: resting motor threshold; s.o.: stimulator's output; SICl: short-interval intracortical inhibition; SICF: short-interval intracortical facilitation; pre Active and post Active, pre Sham and post Sham: before and after repetitive peripheral stimulation in the active and sham sessions (means \pm standard deviations). The asterisk indicates a significant difference $(p \leq 0.05)$.

\section{Resting motor threshold}

In patients, there were no significant effects of SESSION (active or sham SS), TIME (before or after stimulation) or interaction SESSION*TIME $(\mathrm{p}>0.05)$ in regard to rMT.

In controls, there was a significant effect of SESSION $(\mathrm{F}=7.2 ; \mathrm{p}=0.044)$, without significant effect of TIME or interaction SESSION*TIME. Post-hoc analysis showed a significant increase in rMT after active $(\mathrm{p}=0.013)$, but not after sham SS $(\mathrm{p}=0.526)$.

\section{MEP/M ratios (normalized MEP amplitudes)}

Figure 2 shows results in the active and control groups. Data from one subject were excluded due to technical factors.

In controls, there was a significant interaction of SESSION, TIME and INTENSITY (rMT, 130\% rMT, and $100 \%$ of the stimulator's output (s.o.); $\mathrm{F}=6.7 ; \mathrm{p}=0.03$ ). As expected, MEP/M ratios were greater at higher stimulation intensities. Post-hoc analysis showed a significant increase in $\mathrm{MEP} / \mathrm{M}$ ratios at the intensity of $100 \%$ of the s.o. after active $(\mathrm{p}=0.028)$, but not after sham SS $(\mathrm{p}=0.452)$. There were no significant changes at the other two intensities of stimulation $(\mathrm{p}>0.05)$.

In patients, there was only a significant effect of INTENSITY $(\mathrm{F}=10.7, \mathrm{p}=0.009)$, with no significant effects of TIME, SESSION or any other significant interactions $(p>0.05)$.

\section{$\mathrm{SICl}$ and SICF}

There were no significant effects of SESSION or TIME, nor interactions SESSION x TIME for SICI or SICF, in either group after active or sham SS ( $p>0.05)$.

\section{Discussion}

The main result of this preliminary study was the increase in MEP/M ratios after SS in controls but not in patients with unilateral cerebellar infarcts in the chronic phase, in line with results obtained in animals at an acute stage after downregulation of cerebellar outputs $[1,2]$. This result supports the hypothesis that normal cerebellar activity is required so that SS can modulate excitability of the sensorimotor cortex.

SS inputs reach the sensorimotor cortex by thalamocortical pathways, and reach the cerebellum by the spinocerebellar and spinoolivocerebellar tracts. The capacity of the cerebellum to process sensory information is underscored by estimates that, for each efferent axon that leaves the cerebellum, there are more than 40 afferent axons [9]. Cerebellar outputs project to the primary sensorimotor cortex mainly through the cerebellothalamocortical pathway.

It has been hypothesized that SS in the form of repetitive nerve stimulation, as performed in the present study, 
Table 2 Characteristics of patients with cerebellar infarcts

\begin{tabular}{llllllll}
\hline Patient & Age $(\mathbf{y})$ & Gender & Handedness & SARA & NIHSS & Arterial territory & Stimulus intensity (V)* \\
\hline 1 & 63 & F & R & 1 & 0 & PICA & 70 \\
2 & 49 & M & R & 6 & 2 & PICA & 80 \\
3 & 39 & F & L & 11 & 4 & SUCA & 20 \\
4 & 56 & M & R & 1 & 0 & PICA & 40 \\
5 & 48 & M & R & 4.5 & 0 & PICA & 60 \\
6 & 41 & M & R & 0 & 0 & PICA & 70 \\
7 & 49 & M & R & 2 & 2 & SUCA & 50 \\
8 & 21 & F & R & 1 & 0 & PICA & 4,5 \\
9 & 55 & M & R & 0 & 0 & PICA & 20 \\
10 & 53 & M & R & 9.5 & 3 & PICA & 70 \\
\hline
\end{tabular}

$y=$ years; $F=$ female; $M=$ male; $R$ = right; $L=$ left; SARA = scale for the assessment and rating of ataxia; NIHSS = National Institutes of Health Stroke Scale; PICA = posterior inferior cerebellar artery; SUCA = superior cerebellar artery; ${ }^{*}$ initial stimulus intensity in the active somatosensory stimulation session (see text); $V=$ Volts.

may enhance cortical excitability by augmenting effectiveness of the thalamocortical pathway [10]. It has also been suggested that, in order for this phenomenon to occur, functional integrity of cerebellothalamocortical projections is required. Integrity of axons originated in the interpositus nucleus is considered particularly important $[1,3]$.

This concept has been strengthened by animal studies. In rodents, $\mathrm{SS}$ in the form of sciatic nerve stimulation at $10 \mathrm{~Hz}$ for one [1] or two [3] hours enhances motor potentials evoked by cortical electrical or magnetic stimulation of the motor cortex. Ipsilateral cerebellar intranuclear administration of alcohol, the sodium channel blocker tetrodotoxin, or electrical stimulation of cerebellar nuclei impair the enhancement of excitability by SS. In addition, modulation of primary sensorimotor cortex excitability by conditioning SS inputs is diminished in the acute fase after hemicerebellectomy in rats [4].

In this preliminary study, SS in the form of 2-h active repetitive median nerve electrical stimulation did not lead to significant changes in excitability to TMS in

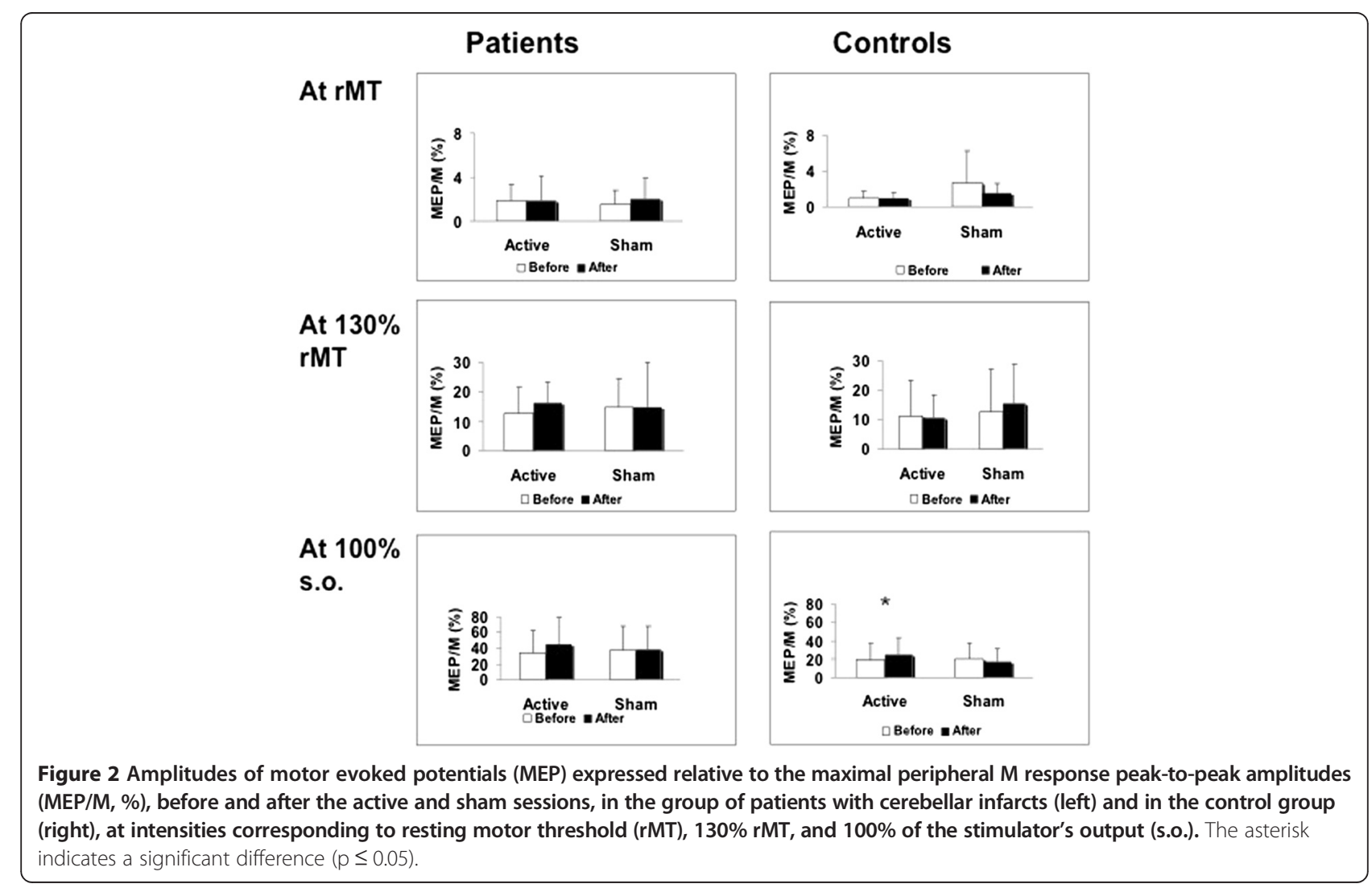


subjects with cerebellar infarcts, but was associated with significant increase in normalized MEP amplitudes from thenar muscles in controls, compared to sham stimulation.

Our patients were in the chronic phase ( $>4$ months) after cerebellar infarcts. Because it has been described that corticomotor excitability to TMS is dynamic over time after a cerebellar infarct [11-13], it remains to be determined whether the observed effect may also be present at early stages after stroke, and whether it becomes more or less intense in the subsiding weeks and months, when responsiveness to cerebellar inputs may undergo plastic changes in the cortex or thalamus.

The increase in normalized MEP amplitudes was observed only at stimulus intensities corresponding to $100 \%$ of the s.o. Mechanisms underlying this phenomenon are not entirely clear. The increase in normalized MEP amplitudes of hand muscles at greater TMS intensities may be explained by recruitment of additional cortical neurons or spinal motor neurons, by evocation of multiple components of the corticospinal volley, or by enhancement of synchronization of spinal motor neurons [14].

In a previous study in healthy subjects, SS in the form of 2-h ulnar nerve stimulation led to increased normalized amplitudes of MEPs recorded at 120\% rMT [5]. MEPs were recorded before and after active SS, and sham stimulation was not performed. In another study in which both active ulnar nerve stimulation and sham SS were applied, enhancement of normalized MEP amplitudes after active SS was more pronounced at greater TMS intensities, in line with our results [6]. Normalized MEPs were recorded before and after both interventions at TMS intensities ranging from below rMT up to $200 \%$ rMT [6]. Increase in normalized MEP amplitudes was noticed in $8 / 10$ subjects at a TMS intensity of $140 \%$ rMT, but not at intensities of $130 \%$ rMT or lower. It is known that MEP amplitudes are less variable/more stable when recorded at greater TMS intensities [15]. We hypothesize that lower variability in MEP amplitudes recorded at higher TMS intensities is associated with greater power to detect changes in normalized MEP amplitudes after interventions such as SS, compared to MEP amplitudes registered at lower TMS intensities.

In addition, in contrast with studies that did not report changes in rMT after 2 h-ulnar nerve stimulation [5,6], in our control group there was a slight increase in rMT after the active session, but not after the sham session. It is possible that technical factors may explain these discrepancies: stimulated nerves (median versus ulnar), target muscles (abductor digiti brevis, ADM, versus thenar muscles) and sham intervention (no stimulation versus stimulation below sensory threshold). rMT seems to reflect membrane excitability and local density of a central core of excitatory interneurons and corticospinal neurons (targeted at the "hot spot"), and also of small spinal cord neurons [16]. MEP/M ratios reflect the extent of activation of the spinal motor neuron pool by a single TMS pulse at a given stimulus intensity [17]. Increases in MEP/M ratios at greater intensities of stimulation reflect progressive recruitment of less excitable or surrounding neurons in relation to the "hot spot" [16].

Even though under some circumstances, increase in rMT are accompanied by decrease in MEP/M ratios for a given target muscle (for instance, after lesions of the motor cortex or the corticospinal tract), interventions that modulate MEP amplitudes may not affect rMT and vice-versa [18]. It has been argued that modulation of MEP amplitudes by sensory input may reflect synaptic plasticity (i.e., lasting enhancement of synaptic transmission through long-term potentiation or long-term depressionlike mechanisms) while changes in rMT would reflect intrinsic plasticity (i.e., excitability mediated by alterations in conductances of membrane ion channels) [18-20]. The increase in $\mathrm{rMT}$ and decrease in MEP/M at $100 \% \mathrm{rMT}$ in the present study may reflect different effects of SS in the form of median nerve stimulation on core and surrounding neurons in the sensorimotor cortex, or different effects of SS on intrinsic and synaptic plasticity. This hypothesis deserves further investigation in a larger sample of subjects.

The lack of modulation of SICI or SICF by SS in the form of median nerve stimulation is consistent with results obtained after ulnar nerve stimulation in healthy subjects [6]. Conversely, in patients with hemiparesis caused by single subcortical strokes, 2-h ulnar and median nerve stimulation was associated with decrease in SICI [21].

The main limitation of this study is its sample size, leading to insufficient power to evaluate between-group differences. In addition, we cannot exclude the possibility that imbalance in gender explains the difference in responsiveness to active SS, compared to sham SS. This is a hypothesis-generating study. Considering the large number of patients screened, we suggest that multicenter studies are needed to confirm our findings.

\section{Conclusions}

In summary, these preliminary results suggest for the first time in humans that cerebellar activity is crucial for modulation of the motor cortex by afferent SS in the form of repetitive peripheral nerve stimulation. These findings are consistent with those reported in rodents and highlight the importance of interactions between somatosensory and cerebellar inputs in modulation of cortical excitability.

\section{Methods}

\section{Subjects}

Ten patients with cerebellar infarcts (Patient group $_{\text {) }}$ and

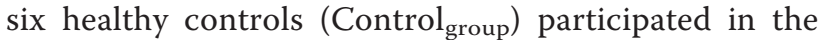
study. 
Inclusion criteria for patients were: age, 18-80 years; first-ever unilateral cerebellar infarct $>4$ months, confirmed by computed tomography or magnetic resonance imaging of the head and without brain lesions in the frontal lobe, parietal lobe, brainstem, thalamus, contralateral cerebellar hemisphere or any part of the corticospinal tract. We opted to include patients $>4$ months post-stroke in order to avoid dynamic changes in cortical excitability that may occur during earlier phases after cerebellar infarcts [11]. Exclusion Criteria were: other neurological or severe chronic diseases; shoulder pain or severe joint deformity; use of medications that interfere with cortical excitability [22]; contraindications to transcranial magnetic stimulation [23]; inability to understand, provide informed consent, or follow instructions of the study.

Figure 3 shows the protocol's flowchart.

Controls were included if they had no history of neurological disease, had a normal neurological examination, and their age, sex and handedness according to the Edinburgh Inventory [24] were comparable to those of the Patient group. $_{\text {. }}$

Median values of age (range) were 49 years (21-63) in patients and 53.5 years (39-64) in controls. Male/female ratios were $7 / 10$ and $5 / 6$ for patient and control subjects, respectively. Nine patients and six controls were righthanded according to the Edinburgh Inventory [24]. In patients, the median score in the SARA (scale for the assessment and rating of ataxia) was 1.5 (range, 0-11) and in the National Institute of Health stroke scale (NIHSS), 0 (range, 0-4).

SARA is an eight-item reliable and valid clinical scale used to assess ataxia; scores range from 0 (no ataxia) to 40 (most severe ataxia) [25]. NIHSS is a widely clinical scale that provides a quantitative measure of strokerelated neurologic deficit. Scores range from 0 to 42 . Higher scores reflects more severe strokes [26].

The project was approved by our institutional Ethics Committee and all subjects provided written consent to participate.

\section{Experimental paradigm}

Figure 1 shows the experimental protocol. This was a singleblind, randomized crossover, placebo-controlled proof-ofprinciple trial. Each subject participated to two sessions of active or sham SS. Each session lasted for about four hours (two hours of SS, and one hour of TMS measurements before and after SS). The order of the sessions was pseudorandomized across subjects. There was an interval of at least six days between the two sessions.

\section{Somatosensory stimulation (SS)}

Each subject sat comfortably in an armchair with eyes open and arms at rest. Two silver surface electrodes

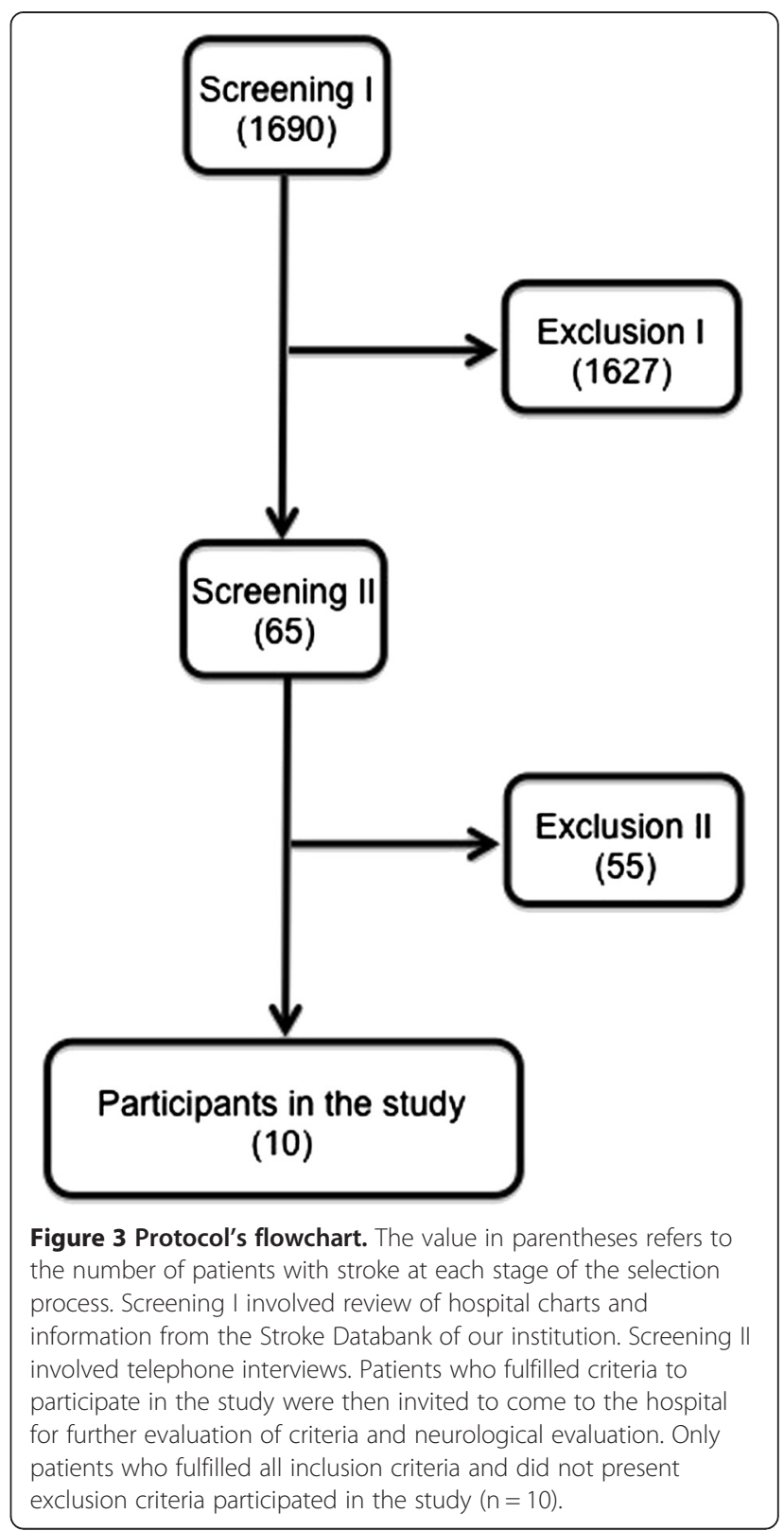

connected to a stimulator were placed on the optimum point for stimulation of the median nerve on the wrist of the affected upper limb, with the cathode placed proximally as previously described $[27,28]$.

Initially, the minimum intensity of stimulation at which patients reported paresthesias in the median nerve cutaneous territory (sensory threshold, ST) was measured three times. Trains of electrical stimuli of $1 \mathrm{~ms}$ duration (frequency of pulses within the train: $10 \mathrm{~Hz}$ ) were administered at $1 \mathrm{~Hz}$ for a period of two hours with a portable stimulator (Alfamedic Ltda., São Paulo, Brazil). The output of the stimulator ranges from 0 to $400 \mathrm{~V}$.

In the active SS session, stimulus intensity was increased until the maximum at which patients reported 
strong paresthesias in the median nerve territory in the absence of pain, while compound muscle action potential amplitudes were below $100 \mu \mathrm{V}$ in the abductor pollicis brevis muscle (APB). Background electromyography (EMG) activity recorded from surface electrodes in the APB was continuously monitored. During the 2-h period, sensations described by patients were checked every five minutes, because they can change at the same stimulus intensity, for example due to changes in skin impedance. If paresthesias decreased or if EMG activity above100 $\mu \mathrm{V}$ was noticed in the APB, stimulus intensities were adjusted accordingly throughout the 2 -h period.

In the sham SS session, intensity was set to $10 \mathrm{~V}$ below sensory threshold $[27,29]$.

\section{Transcranial magnetic stimulation}

TMS was delivered through a figure-of-eight shaped magnetic coil (outside diameter $70 \mathrm{~mm}$, maximum rate of change $22.5 \times 103 \mathrm{~T} / \mathrm{s}$ ) connected to two $200^{2}$ Magstim stimulators through a Bistim ${ }^{2}$ module (The Magstim Company, Dyfed, UK). The magnetic coil was placed tangentially to the scalp, with the intersection of both wings at a $45^{\circ}$ angle with the midline to optimally stimulate the motor cortex [30]. Electromyographic (EMG) activity was recorded from surface electrodes placed over the APB. EMG responses were amplified (1000), filtered (2 Hz-2 kHz) and recorded on a computerized data acquisition system built with the LabVIEW graphical programming language (sampling rate $5 \mathrm{kHz}$ ) [31]. Its conditional triggering' feature was used to deliver TMS stimuli only when the APB was relaxed. Relaxation was defined as EMG activity at baseline $<50$ microvolts $(\mu \mathrm{V})$ peak-to-peak amplitude for at least $1 \mathrm{~s}$.

TMS measurements were obtained after identification of the optimal position (hot spot) of the APB muscle. To define the APB hot spot, we initially set the intensity to $50 \%$ of the maximum stimulator's output, and then decreased it in 5\% steps, moving the coil anteroposteriorly and mediolaterally in steps of $0.5 \mathrm{~cm}$. After identification of points at which no MEPs higher than $100 \mu \mathrm{V}$ were elicited in all directions, the intensity was decreased in 1-2\% steps. The search was repeated iteratively until 3 MEPs were observed out of 3 trials at a given position, while stimulation of adjacent positions did not evoke reliable MEPs on 3 trials. If no MEPs were evoked at any position at a given intensity, while at an intensity $1 \%$ higher, 3 MEPs were still observed out of 3 trials in more than one point, the 'hot spot' was defined as the position in which the largest mean MEP amplitude was detected.

In patients, the cerebral hemisphere contralateral to the cerebellar infarct was stimulated. The homologous hemisphere was stimulated in controls.
The following TMS measurements were performed before and after active or sham SS:

- Resting motor threshold (rMT), defined as the minimum TMS intensity required to elicit at least three out of six motor evoked potentials (MEP) $\geq 50 \mu \mathrm{V}$ in consecutive trials at rest. TMS stimulus intensities were expressed relative to rMT measured from the APB [32].

- MEP amplitudes recorded at intensities corresponding to rMT, $130 \%$ rMT, and 100\% (maximal intensity) of the stimulator's output. Ten trials were recorded at each stimulation intensity. $M$ responses were obtained by supramaximal stimulation of the median nerve at the wrist (10 trials). MEP amplitudes were expressed relative to the maximal peripheral $\mathrm{M}$ response peak-to-peak amplitudes (MEP/M, \%). This measurement controls for differences in muscle bulk and electrode position across subjects and reflects the extent of activation of the spinal motor neuron pool of a target muscle by a single TMS pulse at a given stimulus intensity $[15,17]$.

- Short-interval intracortical facilitation (SICF), shortinterval intracortical inhibition (SICI), determined with a paired-pulse protocol [33]. The intensity of the test stimulus (TS) was that required to evoke MEPs $\left(\mathrm{MEP}_{\mathrm{TS}}\right)$ of approximately $0.5-1 \mathrm{mV}$. The intensity of the conditioning stimulus was $80 \%$ of the APB rMT. The order of presentation of inhibitory (2 ms) and facilitatory (10 ms) trials as well as test stimuli alone was randomized. Sixteen trials were recorded for each interstimulus interval $(2 \mathrm{~ms}$ and $10 \mathrm{~ms}$ ), and sixteen trials were recorded after administration of TS alone.

\section{Statistical analysis}

Repeated-measures analysis of variance was performed separately for the two groups, with factors SESSION (active or sham) and TIME (before and after stimulation). For MEP/M ratios, INTENSITY OF STIMULATION was an additional factor. Post-hoc t-tests were not corrected for multiple comparisons, given the exploratory nature of this study. Mann-Whitney tests were used to compare initial stimulation intensities between the two groups.

\section{Abbreviations}

APB: Abductor pollicis brevis muscle; EMG: Electromyography; MEP: Motor evoked potentials; MEP/M ratios: Amplitudes of motor evoked potentials normalized to amplitudes of supramaximal M responses; $\mu \mathrm{V}$ : Microvolts; NIHSS: National Institutes of Health stroke scale; rMT: Resting motor threshold; SARA: Scale for the assessment and rating of ataxia; SICl: Short-interval intracortical inhibition; SICF: Short-interval intracortical facilitation; SS: Somatosensory stimulation; s.O.: Stimulator's output; TMS: Transcranial magnetic stimulation; TS: Test stimulus; V: Volts.

\section{Competing interests}

The authors declare that they have no competing interests. 


\section{Authors 'contributions}

SNFG participated in data collection, data analysis and was involved in drafting the manuscript. $A B C$ contributed to conception and design of the study, data analysis and interpretation, as well as writing the manuscript. Both authors read and approved the final manuscript.

\section{Acknowledgements}

The authors thank Mr. Ariosto Farias Jr for his substantial contribution in recrutiment.

\section{Author details}

${ }^{1}$ Hospital das Clínicas/São Paulo University, São Paulo, Brazil. ${ }^{2}$ Hospital São Rafael, Salvador, Brazil. ${ }^{3}$ Instituto Israelita de Ensino e Pesquisa Albert Einstein, São Paulo, Brazil. ${ }^{4}$ Rua Waldemar Falcão n 1547 ap 1201 Horto Florestal 40.295-010, Salvador, Bahia, Brazil.

Received: 9 May 2014 Accepted: 5 September 2014

Published: 5 November 2014

\section{References}

1. Taib NOB, Manto M, Laute MA, Brotchi J: The cerebellum modulates rodent cortical motor output after repetitive somato-sensory stimulation. Neurosurgery 2005, 56:811-820

2. Taib NOB, Manto M, Massimo P, Brotchi J: Hemicerebellectomy blocks the enhancement of cortical motor output associated with repetitive somatosensory stimulation in the rat. J Physiol 2005, 567:293-300.

3. Luft AR, Manto MU, Taib NOB: Modulation of motor cortex excitability by sustained peripheral stimulation: the interaction between the motor cortex and the cerebellum. Cerebellum 2005, 4:90-96.

4. Taib NOB, Manto M: Effects of trains of high-frequency stimulation of the premotor/supplementary motor area on conditioned corticomotor responses in hemicerebellectomized rats. Exp Neurol 2008, 212:157-165.

5. Ridding MC, Brouwer B, Miles TS, Pitcher JB, Thompson PD: Changes in muscle responses to stimulation of the motor cortex induced by peripheral nerve stimulation in human. Exp Brain Res 2000, 131:135-143.

6. Kaelin-Lang A, Luft AR, Sawaki L, Burstein AH, Sohn YH, Cohen LG: Modulation of human corticomotor excitability by somatosensory input. J Physiol 2002, 540:622-633.

7. Luft A, Kaelin-Lang A, Hauser TK, Buitrago MM, Thakor NV, Hanley DF, Cohen LG: Modulation of rodent cortical motor excitability by somatosensory input. Exp Brain Res 2002, 142:562-569.

8. Wu CW-H, Gelderen PV, Hanakawa T, Yaseen Z, Cohen LG: Enduring representational plasticity after somatosensory stimulation. Neuroimage 2005, 27:872-884.

9. Heidary $\mathrm{H}$, Tomasch J: Neuron numbers and perikaryon areas in the human cerebellar nuclei. Acta Anat (Basel) 1969, 74:290-296.

10. Meftah EM, Rispal-Padel L: Synaptic plasticity in the thalamo-cortical pathway as one of the neurobiological correlates of forelimb flexion conditioning: Electrophysiological investigation in the cat. J Neurophysiol 1994, 72:2631-2647

11. Liepert J, Kucinski T, Tüscher O, Pawlas F, Bäumer T, Weiller C: Motor cortex excitability after cerebellar infarction. Stroke 2004, 35:2484-2488

12. Da Guarda SNF, Cohen LG, Pinho MC, Yamamoto FI, Marchiori PE, Scaff M, Conforto AB: Interhemispheric asymmetry of corticomotor excitability after chronic cerebellar infarcts. Cerebellum 2010, 9:398-404.

13. Huynh W, Krishnan AV, Vucic S, Lin CS, Kiernan MC: Motor cortex excitability in acute cerebellar infarct. Cerebellum 2013, 12:826-834

14. Devanne H, Lavoie BA, Capaday C: Input-output properties and gain changes in the human corticospinal pathway. Exp Brain Res 1997. 114:329-338.

15. Weber M, Eisen AA: Magnetic stimulation of the central and peripheral nervous systems. Muscle Nerve 2002, 25:160-175.

16. Rossini PM, Rossi S: Transcranial magnetic stimulation: Diagnostic, therapeutic, and research potential. Neurology 2007, 68:484-488.

17. Reis J, Swayne OB, Vandermeeren Y, Camus M, Dimyan MA, Harris-Love M, Perez MA, Ragert P, Rothwell JC, Cohen LG: Contribution of transcranial magnetic stimulation to the understanding of cortical mechanisms involved in motor control. J Physiol 2008, 586:325-351.

18. Delvendahl I, Jung NH, Kuhnke NG, Ziemann U, Mall V: Plasticity of motor threshold and motor-evoked potential amplitude - A model of intrinsic and synaptic plasticity in human motor cortex? Brain Stimul 2012, 5:586-593.
19. Zhang W, Linden DJ: The other side of the engram: experience-driven changes in neuronal intrinsic excitability. Nat Rev Neurosci 2003, 4:885-900.

20. Campanac E, Debanne D: Spike timing-dependent plasticity: a learning rule for dendritic integration in rat CA1 pyramidal neurons. J Physio 2008, 586:779-793

21. Celnik P, Hummel F, Harris-Love M, Wolk R, Cohen LG: Somatosensory stimulation enhances the effects of training functional hand tasks in patients with chronic stroke. Arch Phys Med Rehabil 2007, 88:1369-1376.

22. Ziemann U: TMS and drugs. Clin Neurophysiol 2004, 115:1717-1729.

23. Rossi S, Hallett M, Rossini PM, Pascual-Leone A: Safety, ethical considerations, and application guidelines for the use of transcranial magnetic stimulation in clinical practice and research. Clin Neurophysiol 2009, 120:2008-2039.

24. Oldfield RC: The assessment and analysis of handedness: the Edinburgh inventory. Neuropsychologia 1971, 91:97-113.

25. Brott T, Adams HP Jr, Olinger CP, Marler JR, Barsan WG, Biller J, Spilker J, Holleran R, Eberle R, Hertzberg V, Rorick M, Moomaw CJ, Walker M: Measurements of acute cerebral infarction: a clinical examination scale. Stroke 1989, 20:864-870.

26. Schmitz-Hübsch T, Tezenas du Montcel S, Baliko L, Berciano J, Boesch S, Depondt C, Giunti P, Globas C, Infante J, Kang JS, Kremer B, Mariotti C, Melegh B, Pandolfo M, Rakowicz M, Ribai P, Rola R, Scho“'Is L, Szymanski S, van de Warrenburg BP, Dürr A, Klockgether T: Scale for the assessment and rating of ataxia. Development of a new clinical scale. Neurology 2006, 66:1717-1720.

27. Conforto AB, Cohen LG, dos Santos RL, Scaff M, Marie SKN: Effects of somatosensory stimulation on motor function in chronic cortico-subcortical strokes. J Neurol 2007, 254:333-339.

28. Conforto $A B$, Ferreiro KN, Tomasi $C$, dos Santos $R L$, Moreira VL, Marie SKN, Baltieri SC, Scaff M, Cohen LG: Effects of somatosensory stimulation on motor function after subacute stroke. Neurorehabil Neural Repair 2010, 24:263-272

29. Conforto AB, Kaelin-Lang A, Cohen LG: Increase in hand muscle strength of stroke patients after somatosensory stimulation. Ann Neurol 2002, 51:122-125.

30. Brasil-Neto JP, Cohen LG, Panizza M, Nilsson J, Roth BJ, Hallett M: Optimal focal transcranial magnetic activation of the human motor cortex: effects of coil orientation, shape of the induced current pulse, and stimulus intensity. J Clin Neurophysiol 1992, 9:132-136.

31. Kaelin-Lang A, Cohen LG: Enhancing the quality of studies using transcranial magnetic and electrical stimulation with a new computer-controlled system. J Neurosci Methods 2000, 102:81-89.

32. Hanajima R, Wang R, Nakatani-Enomoto S, Hamada M, Terao Y, Furubayashi T, Okabe S, Inomata-Terada S, Yugeta A, Rothwell JC, Ugawa Y: Comparison of different methods for estimating motor threshold with transcranial magnetic stimulation. Clin Neurophysio/ 2007, 118:2120-2122.

33. Kujirai T, Caramia MD, Rothwell JC, Day BL, Thompson PD, Ferbert A, Wroe S, Asselman P, Marsden CD: Corticortical inhibition in human motor cortex. J Physiol (Lond) 1993, 471:501-519.

doi:10.1186/s40673-014-0016-5

Cite this article as: Farias da Guarda and Conforto: Effects of somatosensory stimulation on corticomotor excitability in patients with unilateral cerebellar infarcts and healthy subjects - preliminary results. Cerebellum \& Ataxias 2016 1:16.

\section{Submit your next manuscript to BioMed Central and take full advantage of:}

- Convenient online submission

- Thorough peer review

- No space constraints or color figure charges

- Immediate publication on acceptance

- Inclusion in PubMed, CAS, Scopus and Google Scholar

- Research which is freely available for redistribution 